\title{
Challenges of Genomic Testing for Hereditary Breast and Ovarian Cancers
}

This article was published in the following Dove Press journal:

The Application of Clinical Genetics

\author{
Lindsey McAlarnen (D) \\ Kristen Stearns (D) \\ Denise Uyar \\ Division of Gynecologic Oncology, \\ Department of Obstetrics and \\ Gynecology, Medical College of \\ Wisconsin, Milwaukee, WI, USA
}

Correspondence: Denise Uyar Division of Gynecologic Oncology, Department of Obstetrics and Gynecology, Medical College of

Wisconsin, 9200 W. Wisconsin Avenue,

Milwaukee, WI 53226, USA

Tel + | 4|4-805-6606

Fax + 4|4-805-6622

Email duyar@mcw.edu

\begin{abstract}
Completion of genetic testing is increasingly important for the complex care of patients with suspected hereditary breast and ovarian cancers (HBOC) and their at-risk family members. Identification of individuals with pathogenic variants has implications for targeted treatment recommendations, risk reduction strategies, increased surveillance recommendations, as well as the genetic testing of family members, known as cascade testing or screening. Due to advances in technology and decreasing costs, what was once single-gene genetic testing has evolved into large-scale multi-gene panel genomic testing. As germline genomic testing for HBOC becomes more and more available, it is important to identify the challenges that are associated with its use. In this manuscript, we review the current issues faced by germline genomic testing for HBOC which include effectively managing the marked increases in genetic referrals, interpreting the vast amount of information yielded by newer testing methods such as next generation sequencing (NGS), recognizing the need for better cascade screening strategies, potential exacerbation of health disparities and improving support for patients navigating the emotional impact related to positive, negative and indeterminate testing results.
\end{abstract}

Keywords: genetic testing, genomic testing, hereditary breast and ovarian cancer syndrome, BRCA, cascade testing, next-generation sequencing

\section{Introduction}

The National Comprehensive Cancer Network (NCCN) and organizations such as the Society of Gynecologic Oncology (SGO) have published recommendations to assist clinicians in the identification of women with hereditary cancer syndromes. ${ }^{1,2}$ Hereditary cancers caused by germline pathogenic variants are responsible for up to $9 \%$ of breast cancers ${ }^{3}$ and up to $20-25 \%$ of all ovarian, fallopian tube, and primary peritoneal cancer cases. ${ }^{4}$ There are multiple genes implicated that may increase the risk for developing breast and/or ovarian cancer. Guidelines state that patients with suspected hereditary breast cancer and all women with epithelial ovarian cancer should receive genetic counseling and be offered comprehensive genetic testing. In settings with appropriate resources, patients are referred to genetic counselors and a comprehensive risk assessment is performed prior to genetic testing.

HBOC is a well-known cancer syndrome where deleterious $B R C A$ variants account for up to $80 \%$ of the pathogenic variants. ${ }^{5}$ Additional high-risk genes $(P A L B 2, T P 53, P T E N)^{1,6}$ or moderate-risk genes $(A T M, C H E K 2)^{1}$ are also associated with HBOC syndrome. Individuals with HBOC syndrome have an elevated lifetime risk of developing ovarian cancer $(40 \%$ and $18 \%$ by age 70 for BRCA1 and 
$B R C A 2$, respectively) and/or breast cancer (57\% and $49 \%$ by age 70 for $B R C A 1$ and $B R C A 2$, respectively). ${ }^{7,8}$ Genetic testing is now commonly recommended at the time of cancer diagnosis and has the potential to impact treatment-related decisions. For example, current guidelines recommend the use of poly ADP-Ribose polymerase inhibitors (PARPi) in different treatment strategies for patients with $B R C A \quad 1 / 2$ associated cancers. Identification of pathogenic variant carriers and at-risk individuals may ultimately reduce the morbidity and mortality from cancer. Genetic testing of at-risk individuals with identification of pathogenic variants before a diagnosis of cancer can significantly impact the trajectory of disease for individuals by affording them the opportunity to consider riskreducing strategies, such as advanced surveillance diagnostics, therapeutics, or surgical interventions.

The process starts with a discussion about genetic counseling and testing followed by informed consent. This process typically involves the patient, their physician, and a genetic counselor. ${ }^{9}$ Previously documented referral rates to genetics for ovarian cancer patients were on the order of $10-30 \% .{ }^{10-12}$ Several studies from just a few years ago found that genetic testing was underutilized with fewer than 1 in 5 individuals with a history of breast or ovarian cancer meeting NCCN criteria undergoing genetic testing. ${ }^{13-16}$ However, since the mapping of the human genome was completed in 2003, medicine has witnessed the birth of what is known as precision medicine: an approach to disease treatment and prevention that seeks to maximize effectiveness by taking into account individual variability in genes, environment, and lifestyle. ${ }^{17}$ Since the human genome mapping milestone, the speed and accuracy of genetic testing have undergone a revolution thanks to technological advances, such as next-generation sequencing, that have made genomic testing faster, more widely available and also managed to decrease associated costs. Genetic testing previously tested individuals for variants in single genes. Modern genetic testing now includes largescale multi-gene panel testing (some panels including up to 80 genes), that generates large amounts of data and is more accurately described as genomic testing.

Completion of genetic and genomic testing is a complicated task with multiple barriers described in the literature: psychosocial factors, logistical challenges, cost implications, poor access to genetic counseling services, low provider recommendation, and limited patient health literacy regarding genetic information. ${ }^{18-20}$ The focus of this review is to explore the impact of genomic testing on patients and patient care models. The challenges associated with genomic testing, although much less studied, are extremely relevant to the care of cancer patients. With more widely available genomic testing, additional obstacles must be considered such as the provision of genetic services amidst steeply rising referrals, interpretation of complicated data given greater numbers of sequence variants, increasing cascade testing in at-risk family members in order to improve patient outcomes, cost effectiveness and disease prevention; recognition of the potential for worsening of current cancer disparities, coping with the psychosocial impact of both positive and negative genetic testing results, and understanding that greater awareness of genetic testing is prompting even younger patients to pursue testing. As more patients complete genomic testing as part of the standard of care, it will become increasingly important to understand these issues and how they may impact patient care in the age of precision medicine.

\section{Provision of Genetic Services}

The Workforce Working Group (WFWG), comprised of US professional genetic counseling organizations, determined that a shortage of genetic counselors is present and could persist until 2030. ${ }^{21}$ Currently, institutions are faced with the challenge of meeting an increased demand for in-person genetic services that exceeds availability and will likely continue into the near future. ${ }^{22}$ In an attempt to address this gap, several studies have evaluated alternatives to inperson genetic counseling services, such as telephone or video conferencing. Cancer genetics is unique in that the initial consultation and testing can be done via telemedicine, with the saliva sample kit sent to the patient's home. What began as a strategy targeting patients in rural areas or for whom travel to the clinic was difficult has evolved into a promising strategy for providing genetics services and expertise in many clinical situations. In a randomized trial comparing tele-genetics to in-person genetic counseling, there was no difference in patient satisfaction, but inperson sessions had higher attendance. ${ }^{23}$ Additionally, telegenetics costs averaged \$106 per session compared to \$244 for an in-patient session. ${ }^{23}$ Additional studies assessing the acceptability of tele-genetics have concluded that live virtual conferencing is well received by patients and providers with the benefit of improved cost effectiveness. ${ }^{24-26}$ Similar to many areas of health care, Coronavirus disease 19 (COVID-19) impacted genetic services, resulting in an acute decrease in genetics referrals. However, it has been 
postulated that the previously established use of telemedicine in genetics likely mitigated this impact. ${ }^{27}$

Another strategy to increase access to genetic services includes expansion of healthcare providers' roles. In one alternative care delivery program, known as mainstreamed genetic testing, additional health care providers take on parts of patient care surrounding genetic services. This was a collaborative approach by oncologists and geneticists, where specially trained oncologists were responsible for patient consenting, testing coordination, and dissemination of results. Individuals with confirmed pathogenic variants or variants of uncertain significance (VUS) were then referred for post-test counseling with a genetic counselor. ${ }^{28}$ This method demonstrated success in streamlining patient care, provided an overall positive patient experience, and demonstrated cost effectiveness. ${ }^{28}$ A larger meta-analysis of 13 studies also concluded that mainstream pathways were both acceptable and feasible. ${ }^{29}$

It is clear from these studies that the growth in the demand for genetic counseling and genetic testing has fostered beneficial innovations in care delivery. Genomic testing is also prompting an examination of the act of counseling and the process of obtaining informed consent. Counseling whether in person or virtually, has been a cornerstone in helping patients understand the complexities of genetic testing, but additional disclosures surrounding genomic testing are essential. Describing differences in the latest test availability and panel testing can be difficult due to differing levels of health literacy. Explaining the concept of genotype as a component of cancer risk, which can be modified by environmental exposures and other factors, and not a definitive predictor of health outcome, is challenging to say the least. ${ }^{30}$ Additionally, large panel testing and the performance of genome-wide sequencing generates large amounts of data with greater chances of encountering unanticipated results. The greater the number of genes included in a panel, the greater the chance of finding genetic variants with unclear medical or cancer risk management recommendations. ${ }^{31}$

\section{Cascade Screening}

In the US, HBOC is estimated to affect over 1 million individuals. ${ }^{32}$ Cascade testing or screening, is the testing of at-risk biologic relatives of individuals who carry a pathogenic variant. The Centers for Disease Control and Prevention (CDC) classified Tier 1 genomic applications for hereditary breast and ovarian cancer syndrome (HBOC), Lynch Syndrome (LS), and familial hyper- cholesterolemia (FH), thus supporting the clinical use of cascade screening specifically for these syndromes. The goals of cascade screening are to identify, inform, and ideally assist in the surveillance of at-risk relatives found to have a pathogenic variant. This is also termed presymptomatic genetic testing. An additional benefit of cascade testing is the potential cost-savings associated with singlesite testing. If a pathogenic mutation is identified in a family, further genetic testing of family members only requires single-site gene testing for the specific gene mutation, rather than the more expensive testing of an entire gene or panel of genes.

Due to the Health Insurance and Portability Act (HIPPA) of 1996, healthcare providers are not permitted to disclose protected health information to relatives. Thus, the process of informing at-risk relatives typically relies on the individual patient diagnosed with the pathogenic variant (proband) providing a copy of their test results along with written information ("family letter"). Distribution of family letters relies on the correct identification of all atrisk family members, the ability of the proband to contact those family members, and the ability of the at-risk family members to understand the information provided to them. Rates of uptake for cascade screening have been undoubtedly disappointing. Studies estimate that the current rates of cascade testing range from $15 \%$ to $57 \%$ when the proband is responsible for contacting their relatives. ${ }^{33}$

Transfer of information from patient to family members is an area in need of improvement. Although some studies endorse family letters as an effective method for dissemination of information, ${ }^{34}$ others have shown this strategy to be ineffective. One study found that while nearly all family members of $B R C A$ positive patients would like to have known their risks, only half of these individuals were aware of the risks of inherited pathogenic variants or knowledgeable about the opportunity to pursue cascade testing. ${ }^{35}$ Additionally, preferences for sharing genetic testing results differ between patients and their family members. Family members seem to prefer direct contact from either a physician or clinic, whereas patients (probands) prefer indirect contact methods (ie, family letters). ${ }^{36,37}$ Roberts et al reviewed a spectrum of cascade testing methodologies in 122 studies. $^{38}$ They concluded that there were no identifiable strategies to date that have optimized cascade testing in hereditary conditions and highlighted that genetic disclosure laws vary by state and may limit cascade screening initiatives. Roberts et al suggests that a public health approach, such as mandatory 
reporting of clinically actionable genetic diseases, bidirectional reporting between registries and medical facilities, and centralized cascade screening conducted by state departments of health, could help facilitate providers' contacting at-risk relatives. ${ }^{38}$ Enhancing resources and support for patients so they can be better prepared to engage in difficult conversations with family members has also been suggested as a strategy to improve cascade screening. ${ }^{39}$ Finding effective cascade screening strategies is an important opportunity for precision medicine to impact individual health, population health, and health systems through disease prevention and represents an urgent need today.

\section{Next-Generation Sequencing and Variants of Unknown Significance}

Genetic variants are classified based on the probability that the altered DNA sequence is pathogenic. ${ }^{40}$ The variant classification system involves classes of benign, likely benign, likely pathogenic, and pathogenic. ${ }^{40,41}$ Assignment of a variant to the correct group allows the proper management of genetic information. An additional group, the largest group with the broadest probability of pathogenicity, is variants of uncertain significance (VUS). ${ }^{40}$ Increasingly, VUS results are being identified by genetic testing due to NGS techniques which sequence hundreds of DNA fragments simultaneously. Due to the location of these variants in noncoding regions or less functionally relevant domains of DNA, the impact of VUS on protein function is more difficult to ascertain compared to known pathogenic variants. ${ }^{41}$ The American College of Medical Genetics and Genomics (ACMG) states that variants of uncertain significance should not be used for clinical decision-making. ${ }^{42}$ This poses a conundrum for genetic counselors and clinicians when a patient receives a VUS result. At times, this leads to a stronger focus on clinical presentation and family history while awaiting reclassification of VUS. ${ }^{43}$ This can also result in incorporation of additional evidence such as functional assays to reclassify a VUS defined previously. ${ }^{44}$ Better defining of VUS result is a current area of investigation, with in vitro functional assays, algorithms, and updated disease databases all contributing to the goal. ${ }^{41}$ The question of who is responsible for recontacting patients as additional information is updated and VUS results become known entities still remains unanswered.

As noted, NGS describes a high throughput massive parallel sequencing method that enables the rapid sequencing of hundreds of DNA or RNA fragments belonging to the human or tumor genome. ${ }^{41,45}$ This technique allows for the analysis of single-gene or multi-gene panels. The use of NGS panel testing provides an efficient and costeffective method for completing genomic testing when multi-gene testing is indicated. NGS has vastly increased the amount of information regarding genetic variants and contributed to patient-specific treatment strategies, ${ }^{41,45}$ such as the utilization of PARP inhibitors for patients with $B R C A$ associated cancers.

Genes on NGS cancer panels may be classified into 3 groups based on penetrance: high risk, moderate risk and low or unknown risk. ${ }^{46}$ The majority of genetic variants do not have specific clinical treatments or risk management guidelines. ${ }^{41,47}$ While the idea of more data would seem to correlate with better outcomes, this has not been shown to be the case. Identification of mutations in low or unknown penetrance genes lack well-established guidelines and have been identified as a source of anxiety for many patients. $^{31}$ At times, the sheer quantity of information provided by NGS leads to more questions than answers, a concept known as the Next-Generation Sequencing Paradox: ${ }^{41}$ the more genes tested in a panel, the greater the likelihood of VUS detection and the greater the ambiguity of the test results. This is easily demonstrated in current clinical testing. The Ambry Genetics Lab currently offers a comprehensive 36-gene panel for hereditary cancer and cites a VUS detection rate of its multi-gene panels at $14 \%$ on its website ${ }^{48}$ compared with single-gene testing VUS rate of $2.1 \%$ (Myriad Genetics Lab). ${ }^{49}$ As testing becomes more sophisticated and panels include increasing numbers of genes, this paradox will continue until more data can be obtained on VUS.

The complexity of NGS data interpretation and classification of variants are real-time challenges for clinical use. $^{41}$ Databases are routinely used to guide geneticists and clinicians in interpretation of genetic data. Such databases can be grouped by population, disease, and sequence. ${ }^{42}$ Database use can be challenging due to the vast amount of information, possibility of incorrect variant classification, and the need for continuous updating of classifications as new evidence about existing variants becomes available. ${ }^{42}$ Using genetic data to treat a single patient can be problematic, and the ACMG recommends against utilizing molecular testing as the sole evidence for disease diagnosis. Rather, clinical and genetic information should be utilized in combination. ${ }^{42}$ For example, when a variant classified as "likely pathogenic" presents with clinical and imaging findings consistent with a specific 
condition, this provides enough evidence to help guide medical decisions. Given the multitude of genetic variants, grouping patients for clinical outcomes analysis based on a single genetic variant requires larger sample sizes than could reasonably be recruited for clinical trial or analysis. $^{47}$ Pooling data on VUS via collaborative datasharing efforts will be essential to the elucidation of VUS result in the US and around the world.

\section{Healthcare Disparities}

The incidence of cancer and cancer mortality is known to vary between ethnic groups. In the US, African Americans have higher death rates than all other racial/ethnic groups for many cancers. ${ }^{50}$ For example, despite having similar rates of breast cancer, African American women are more likely than white women to die of the disease. Similarly, African American men are twice as likely as white men to die of prostate cancer and continue to have the highest prostate cancer mortality among all US population groups. $^{50,51}$ The underlying causes of these findings are multifactorial and only beginning to be understood but demand that greater efforts be directed towards the reversal of these trends.

Although genetic testing has increased in the US, this has not been experienced equally among all racial and ethnic groups. ${ }^{52,53}$ Several studies have reported that awareness significantly differs by ethnicity, and more white participants were aware of cancer risk than Hispanic, African American, or Asian participants. ${ }^{54,55}$ Studies found a greater mistrust among racial and ethnic minorities regarding how genetic information would be used. Lack of confidence in genetic testing results and mistrust regarding the utilization of genetic testing results have been identified as powerful deterrents to engagement in genetic testing for many minority populations. ${ }^{56,57}$ Thus, underutilization of genetic testing has been more pronounced in racial/ethnic minorities. The lack of awareness, understanding of perceived risk, provider recommendation, and equal access to specialized care are themes that have contributed to persistent health disparities in genetic services. ${ }^{58}$ Disproportionate increases in genomic testing means that data on underrepresented groups will continue to be limited and benefits of genomic testing and treatment decisions will not be equally realized among all populations. Data that does not adequately reflect the racial and ethnic diversity in the US limits the generalizability of current genetic/genomic research and the subsequent guideline recommendations that will result may magnify known disparities.

Equal access to genetic testing should be included in the modern standard of care for all patients with suspected hereditary cancer syndromes. Less available information on racial and ethnic minorities translates into less information available for all patients and providers about possible pathogenic variants.

To overcome the lack of diversity in large-scale genomic data, greater efforts need to be directed toward the sharing of clinical and genomic data across health systems, academic institutions, and industry partners.

\section{Psychological Effects of Genetic Testing}

Genetic testing can lead to psychological effects including worry, anxiety, depression, and confusion over one's risk of cancer. $^{59}$ In a review of 21 articles investigating psychological outcomes of pathogenic variant carriers and non-carriers, two categories of psychological effects were examined: distress and anxiety/depression. ${ }^{60}$ The majority of studies reported increased distress, or negative emotional experience interfering with an individual's ability to cope with life events, among carriers of pathogenic variants. ${ }^{60}$ Pathogenic variant carriers have been observed to have higher levels of anxiety and depression when compared to nonpathogenic variant carriers. Interestingly, some studies indicate the peak in this psychological effect seems to return to baseline after 6-12 months, ${ }^{60-62}$ while other studies have had conflicting findings. In a prospective study of 464 women who underwent $B R C A 1 / 2$ testing, pathogenic variant carriers reported significantly more genetic testing distress persisting five years after testing as measured by the Multi-Dimensional Assessment of Cancer Risk Assessment (MICRA), an instrument specifically designed to measure distress associated with a positive $B R C A 1 / 2$ pathogenic variant result, when compared to non-carriers. ${ }^{63}$

Uninformative test results occur when a patient tests negative for pathogenic variants such as $B R C A$ but remain at high risk for disease based on family history. Perceived stress is reported as greater among $B R C A$ pathogenic variant carriers when compared to those with uninformative test results though no differences in clinically significant depression among the two groups was noted. ${ }^{63}$ When $B R C A$ carriers underwent risk reducing bilateral salpingooophorectomy, their perceived risk of cancer appeared to 
decrease, but unfortunately, this did not correspond to a reduction in distress over time. ${ }^{63}$ Additionally, one study identified lower post-testing distress scores among individuals who received a positive genetic testing result when compared to individuals who did not undergo genetic testing at all, but increased distress among patients with VUS results. ${ }^{64}$ Important to note is that racial and ethnic minorities not only have higher rates of ambiguous VUS results but have greater genetic testing specific distress regardless of testing outcome. ${ }^{64}$ Anticipating this distress should be an important part of pre-test counseling.

For those patients with a diagnosis of ovarian cancer, genetic testing for the purposes of guiding therapies can conceivably impact a patient psychologically. PARP inhibitors are currently utilized for frontline maintenance and treatment of recurrence. ${ }^{65}$ While FDA approved indications for PARP inhibitors continue to evolve, patients with deleterious $B R C A$ variants and homologous recombination deficiency currently have more treatment options. The potential to use PARP inhibitors is likely another motivator for patients to undergo genetic testing. Thus, awareness of the implications that genetic testing results might have on available therapeutic options also likely contributes to patient anxiety.

When and how should at risk individuals be informed of their genetic risk is another central theme of research surrounding the impact of genetic testing. It is not recommended that young adults under the age of 18 undergo cascade screening/presymptomatic testing, and most guidelines do not recommend increased surveillance strategies before age 25. However, increasing numbers of individuals are undergoing testing between the ages of 18-24 and may receive results in the absence of defined management strategies. Given the current techniques, they also may have a greater chance of receiving ambiguous VUS results. These young individuals represent an understudied group, but some researchers have begun to identify the separate concerns present for young carriers of pathogenic variants. One study by Werner-Lin et al analyzed data from pooled studies which included women aged 18-24 who had considered or completed genetic testing. ${ }^{66}$ Participants were noted to be very early in the process of understanding their family history of cancer, which can influence their decision-making. This younger cohort also had greater reliance on parents for emotional and financial support, accompanied by a greater sense of urgency in risk management. They more often either intended to pursue or completed risk reducing procedures earlier than anticipated, likely related to having coverage under parents' insurance plans and the undesired anticipation of facing decades of screening. ${ }^{66}$ Younger age is also associated with worse experience scores and MICRA scores indicating poorer psychological adaptation to results. ${ }^{66}$ These findings have been confirmed in additional studies as well. ${ }^{67}$ Emphasis on the longitudinal support needed by young at-risk individuals as they attempt to balance autonomy, genetic knowledge and evolving life circumstances is needed in future research.

\section{Discussion}

Single-gene genetic testing and today's modern genomic testing have had a significant positive impact on the care of patients with hereditary cancer. As genomic testing is likely to become an even greater component of patient care in the future, it will be important for providers to be mindful of emerging concerns associated with genomic testing. Greater awareness, availability and sheer data afforded by genomic testing have enabled cancer care to take a tremendous leap forward ${ }^{41}$ but this has been accompanied by challenges. Testing demands that outpace the current genetic resources, ${ }^{21}$ increased uncertainty for more individuals undergoing genomic testing, ${ }^{41,42}$ disappointing cascade screening efforts, risk of widening current health disparities, ${ }^{50}$ and need for greater psychologic support are among the many challenges faced by patients, at-risk family members and providers. In addition, genomic testing has brought a host of philosophical and ethical issues to the forefront: how does one truly consent patients for an entire panel of genes and all the potential outcomes? Who should be responsible for rechecking and updating patients on the status of VUS result? How should providers navigate the notion of "do no harm" with that of "the duty to warn" when it concerns family members of patients with pathogenic variants across states with variable legislation? How can providers better support those with positive, negative and uninformative genetic test results? How can more resources be devoted to the longer term needs of those individuals diagnosed with pathogenic variants ahead of established guidelines? And perhaps most importantly, will there be equity and representation to raise the level of care for all patients with hereditary cancer regardless of race and ethnicity? In this review, we have identified several genomic testing considerations, but there are surely more to come. One additional aspect, out of scope of this review, is determining the correct place for the now widely available directto-consumer testing. Direct-to-consumer (DTC) testing is a commercial model of genetic testing, marketed directly to 
consumers without the involvement of genetic counselors or healthcare providers. This strategy may seem counterintuitive if we consider, as we just have, the incredible complexities of genomic testing that exist even when trained professionals are involved, and yet the number of companies offering DTC testing is rapidly growing.

\section{Conclusion}

Genomic testing for hereditary cancer syndromes is an increasingly important adjunct to patient care with immense potential that is only beginning to be realized. Understanding and addressing current patient, at-risk family members and provider concerns regarding genomic testing will contribute to improved experiences for all.

\section{Disclosure}

The authors report no conflicts of interest in this work.

\section{References}

1. Daly MB, Pilarski R, Yurgelun MB, et al. NCCN guidelines insights: genetic/familial high-risk assessment: breast, ovarian, and pancreatic, version 1.2020. J Natl Compr Canc Netw. 2020;18(4):380-391. doi:10.6004/jncen.2020.0017

2. Lancaster JM, Powell CB, Chen L-M, et al. Society of gynecologic oncology statement on risk assessment for inherited gynecologic cancer predispositions. Gynecol Oncol. 2015;136(1):3-7. doi:10.1016/j.ygyno.2014.09.009

3. Young SR, Pilarski RT, Donenberg T, et al. The prevalence of BRCA1 mutations among young women with triple-negative breast cancer. BMC Cancer. 2009;9(1):86. doi:10.1186/1471-2407-9-86

4. Walsh T, Casadei S, Lee MK, et al. Mutations in 12 genes for inherited ovarian, fallopian tube, and peritoneal carcinoma identified by massively parallel sequencing. Proc Natl Acad Sci U S A. 2011;108(44):18032-18037. doi:10.1073/pnas.1115052108

5. Kobayashi H, Ohno S, Sasaki Y, et al. Hereditary breast and ovarian cancer susceptibility genes (review). Oncol Rep. 2013;30 (3):1019-1029. doi:10.3892/or.2013.2541

6. Ellsworth RE, Decewicz DJ, Shriver CD, et al. Breast cancer in the personal genomics era. Curr Genomics. 2010;11(3):146-161. doi:10.2174/138920210791110951

7. Chen S, Parmigiani G. Meta-analysis of BRCA1 and BRCA2 penetrance. J Clin Oncol. 2007;25(11):1329-1333. doi:10.1200/ JCO.2006.09.1066

8. Knapke S, Haidle JL, Nagy R, et al. The current state of cancer genetic counseling access and availability. Genet Med. 2016;18 (4):410-412. doi:10.1038/gim.2015.98

9. Robson ME, Bradbury AR, Arun B, et al. American society of clinical oncology policy statement update: genetic and genomic testing for cancer susceptibility. J Clin Oncol. 2015;33(31):3660-3667. doi:10.1200/JCO.2015.63.0996

10. Hayden S, Mange S, Duquette D, et al. Large, prospective analysis of the reasons patients do not pursue BRCA genetic testing following genetic counseling. $J$ Genet Couns. 2017;26(4):859-865. doi:10.1007/s10897-016-0064-5

11. Uyar D, Neary J, Monroe A, et al. Implementation of a quality improvement project for universal genetic testing in women with ovarian cancer. Gynecol Oncol. 2018;149(3):565-569. doi:10.1016/ j.ygyno.2018.03.059
12. Hoskins PJ, Gotlieb WH. Missed therapeutic and prevention opportunities in women with BRCA-mutated epithelial ovarian cancer and their families due to low referral rates for genetic counseling and BRCA testing: a review of the literature. CA Cancer J Clin. 2017;67 (6):493-506. doi:10.3322/caac.21408

13. Childers CP, Childers KK, Maggard-Gibbons M, et al. National estimates of genetic testing in women with a history of breast or ovarian cancer. J Clin Oncol. 2017;35(34):3800-3806. doi:10.1200/ JCO.2017.73.6314

14. Wright JD, Chen L, Tergas AI, et al. Underuse of BRCA testing in patients with breast and ovarian cancer. Am J Obstet Gynecol. 2016;214(6):761-763. doi:10.1016/j.ajog.2016.02.011

15. Randall LM, Pothuri B, Swisher EM, et al. Multi-disciplinary summit on genetics services for women with gynecologic cancers: a society of gynecologic oncology white paper. Gynecol Oncol. 2017;146 (2):217-224. doi:10.1016/j.ygyno.2017.06.002

16. Febbraro T, Robison K, Wilbur JS, et al. Adherence patterns to national comprehensive cancer network ( $\mathrm{NCCN}$ ) guidelines for referral to cancer genetic professionals. Gynecol Oncol. 2015;138 (1):109-114. doi:10.1016/j.ygyno.2015.04.029

17. Precision Medicine Initiative Working Group. The Precision Medicine Initiative Cohort Program - Building a Research Foundation for 21st Century Medicine. 2015.

18. Committee on Gynecologic Practice. ACOG committee opinion no. 727: cascade testing: testing women for known hereditary genetic mutations associated with cancer. Obstet Gynecol. 2018;131(1):e31e34. doi:10.1097/AOG.0000000000002457

19. Hughes KS. Genetic testing: what problem are we trying to solve? J Clin Oncol. 2017;35(34):3789-3791. doi:10.1200/ JCO.2017.74.7899

20. Cornel MC, van El CG. Barriers and facilitating factors for implementation of genetic services: a public health perspective. Front Public Health. 2017;5:195. doi:10.3389/fpubh.2017.00195

21. Hoskovec JM, Bennett RL, Carey ME, et al. Projecting the supply and demand for certified genetic counselors: a workforce study. J Genet Couns. 2018;27(1):16-20. doi:10.1007/s10897017-0158-8

22. Eismann S, Vetter L, Keller M, et al. Long-term experiences with genetic consultation in people with hereditary breast and ovarian cancer. Arch Gynecol Obstet. 2016;294(5):1011-1018. doi:10.1007/ s00404-016-4133-7

23. Buchanan AH, Datta SK, Skinner CS, et al. Randomized trial of telegenetics vs. in-person cancer genetic counseling: cost, patient satisfaction and attendance. J Genet Couns. 2015;24(6):961-970. doi:10.1007/s10897-015-9836-6

24. Zierhut HA, MacFarlane IM, Ahmed Z, et al. Genetic counselors' experiences and interest in telegenetics and remote counseling. J Genet Couns. 2018;27(2):329-338. doi:10.1007/s10897-017-0200$\mathrm{x}$

25. Bradbury A, Patrick-Miller L, Harris D, et al. Utilizing remote real-time videoconferencing to expand access to cancer genetic services in community practices: a multicenter feasibility study. $J$ Med Internet Res. 2016;18(2):e23. doi:10.2196/jmir.4564

26. Hilgart JS, Hayward JA, Coles B, et al. Telegenetics: a systematic review of telemedicine in genetics services. Genet Med. 2012;14:765-776.

27. Yin K, Singh P, Drohan B, et al. Breast imaging, breast surgery, and cancer genetics in the age of COVID-19. Cancer. 2020;126:4466-4472.

28. McLeavy L, Rahman B, Kristeleit R, et al. Mainstreamed genetic testing in ovarian cancer: patient experience of the testing process. Int J Gynecol Cancer. 2020;30(2):221-226. doi:10.1136/ijgc-2019000630

29. Scheinberg T, Young A, Woo H, et al. Mainstream consent programs for genetic counseling in cancer patients: a systematic review. Asia Pac J Clin Oncol. 2020. doi:10.1111/ajco.13334. 
30. Greville-Heygate SL, Eccles DM, Side LE. Hereditary breast and ovarian cancer testing in the genomic era. JAMA Oncol. 2019;5 (1):58. doi:10.1001/jamaoncol.2018.3034

31. Hall MJ, Forman AD, Pilarski R, et al. Gene panel testing for inherited cancer risk. $J$ Natl Compr Canc Netw. 2014;12 (9):1339-1346. doi:10.6004/jncen.2014.0128

32. George R, Kovak K, Cox SL. Aligning policy to promote cascade genetic screening for prevention and early diagnosis of heritable diseases. J Genet Couns. 2015;24(3):388-399. doi:10.1007/s10897014-9805-5

33. Menko FH, Ter Stege JA, van der Kolk LE, et al. The uptake of presymptomatic genetic testing in hereditary breast-ovarian cancer and lynch syndrome: a systematic review of the literature and implications for clinical practice. Fam Cancer. 2019;18(1):127-135. doi:10.1007/s10689-018-0089-z

34. van der Roest WP, Pennings JM, Bakker M, et al. Family letters are an effective way to inform relatives about inherited cardiac disease. Am J Med Genet A. 2009;149A(3):357-363. doi:10.1002/ajmg. a. 32672

35. Sermijn E, Goelen G, Teugels E, et al. The impact of proband mediated information dissemination in families with a BRCA1/2 gene mutation. J Med Genet. 2004;41(3):e23. doi:10.1136/ jmg.2003.011353

36. Sermijn E, Delesie L, Deschepper E, et al. The impact of an interventional counselling procedure in families with a BRCA1/2 gene mutation: efficacy and safety. Fam Cancer. 2016;15(2):155-162. doi:10.1007/s10689-015-9854-4

37. Sturm AC. Cardiovascular cascade genetic testing: exploring the role of direct contact and technology. Front Cardiovasc Med. 2016;3:11. doi: $10.3389 /$ fcvm. 2016.00011

38. Roberts MC, Dotson WD, DeVore CS, et al. Delivery of cascade screening for hereditary conditions: a scoping review of the literature. Aff (Millwood). 2018;37(5):801-808. doi:10.1377/hlthaff.2017.1630

39. Pollard S, Kalloger S, Weymann D, et al. Genetic testing for hereditary cancer syndromes: patient recommendations for improved risk communication. Health Expect. 2020;23(4):884-892. doi:10.1111/ hex.13062

40. Moghadasi S, Eccles DM, Devilee P, et al. Classification and clinical management of variants of uncertain significance in high penetrance cancer predisposition genes. Hum Mutat. 2016;37(4):331-336. doi:10.1002/humu.22956

41. Federici G, Soddu S. Variants of uncertain significance in the era of high-throughput genome sequencing: a lesson from breast and ovary cancers. J Exp Clin Cancer Res. 2020;39(1):46. doi:10.1186/s13046020-01554-6

42. Richards S, Aziz N, Bale S, et al. Standards and guidelines for the interpretation of sequence variants: a joint consensus recommendation of the American college of medical genetics and genomics and the association for molecular pathology. Genet Med. 2015;17 (5):405-424. doi:10.1038/gim.2015.30

43. Chang J, Seng S, Yoo J, et al. Clinical management of patients at risk for hereditary breast cancer with variants of uncertain significance in the era of multigene panel testing. Ann Surg Oncol. 2019;26 (10):3389-3396. doi:10.1245/s10434-019-07595-2

44. Kim H-K, Lee EJ, Lee Y-J, et al. Impact of proactive high-throughput functional assay data on BRCA1 variant interpretation in 3684 patients with breast or ovarian cancer. J Hum Genet. 2020;65 (3):209-220. doi:10.1038/s10038-019-0713-2

45. Shendure J, Ji JH. Next-generation DNA sequencing. Nat Biotechnol. 2008;26(10):1135-1145. doi:10.1038/nbt1486

46. Fecteau H, Vogel KJ, Hanson K, et al. The evolution of cancer risk assessment in the era of next generation sequencing. $J$ Genet Couns. 2014;23(4):633-639. doi:10.1007/s10897-014-9714-7

47. Morash M, Mitchell $\mathrm{H}$, Beltran $\mathrm{H}$, et al. The role of next-generation sequencing in precision medicine: a review of outcomes in oncology. J Pers Med. 2018;8(3):30. doi:10.3390/jpm8030030.
48. VUS genetic testing | ambry genetics. Available from: https://www.ambry gen.com/providers/bringing-clarity. Accessed December 12, 2020.

49. Eggington JM, Bowles KR, Moyes K, et al. A comprehensive laboratory-based program for classification of variants of uncertain significance in hereditary cancer genes. Clin Genet. 2014;86 (3):229-237. doi:10.1111/cge.12315

50. AACR cancer disparities progress report 2020. Philadelphia: American Association for Cancer Research. Available from: http://www.cancerdis paritiesprogressreport.org. Accessed September 27, 2020.

51. Cancer disparities. National cancer institute; 2016. Available from: https://www.cancer.gov/about-cancer/understanding/disparities. Accessed 2020.

52. Mai PL, Vadaparampil ST, Breen N, et al. Awareness of cancer susceptibility genetic testing: the 2000, 2005, and 2010 National health interview surveys. Am J Prev Med. 2014;46(5):440-448. doi:10.1016/j.amepre.2014.01.002

53. Rosenberg SM, Ruddy KJ, Tamimi RM, et al. BRCA1 and BRCA2 mutation testing in young women with breast cancer. JAMA Oncol. 2016;2(6):730-736. doi:10.1001/jamaoncol.2015.5941

54. Adams I, Christopher J, Williams KP, et al. What Black women know and want to know about counseling and testing for BRCA1/2. J Cancer Educ. 2015;30(2):344-352. doi:10.1007/s13187-014-0740-9

55. Sussner KM, Edwards T, Villagra C, et al. BRCA genetic counseling among at-risk Latinas in New York City: new beliefs shape new generation. J Genet Couns. 2015;24(1):134-148. doi:10.1007/ s10897-014-9746-z

56. Allford A, Qureshi N, Barwell J, et al. What hinders minority ethnic access to cancer genetics services and what may help? Eur J Hum. 2014;22(7):866-874. doi:10.1038/ejhg.2013.257

57. Saulsberry K, Terry SF. The need to build trust: a perspective on disparities in genetic testing. Genet Test Mol Biomarkers. 2013;17 (9):647-648. doi:10.1089/gtmb.2013.1548

58. Hall M, Olopade OI. Confronting genetic testing disparities: knowledge is power. JAMA. 2005;293(14):1783-1785. doi:10.1001/ jama.293.14.1783

59. Nelson HD, Pappas M, Cantor A, et al. Risk assessment, genetic counseling, and genetic testing for BRCA-related cancer in women. JAMA. 2019;322(7):666-685. doi:10.1001/jama.2019.8430

60. Lombardi L, Bramanti SM, Babore A, et al. Psychological aspects, risk and protective factors related to BRCA genetic testing: a review of the literature. Support Care Cancer. 2019;27(10):3647-3656. doi:10.1007/s00520-019-04918-7

61. Beran TM, Stanton AL, Kwan L, et al. The trajectory of psychological impact in BRCA1/2 genetic testing: does time heal? Ann Behav Med. 2008;36(2):107-116. doi:10.1007/s12160-008-9060-9

62. Smith AW, Dougall AL, Posluszny DM, et al. Psychological distress and quality of life associated with genetic testing for breast cancer risk. Psychooncology. 2008;17(8):767-773. doi:10.1002/pon.1291

63. Graves KD, Vegella P, Poggi EA, et al. Long-term psychosocial outcomes of BRCA1/BRCA2 testing: differences across affected status and risk-reducing surgery choice. Cancer Epidemiol Biomarkers Prev. 2012;21(3):445-455. doi:10.1158/1055-9965.EPI-11-0991

64. Lumish HS, Steinfeld H, Koval C, et al. Impact of panel gene testing for hereditary breast and ovarian cancer on patients. $J$ Genet Couns. 2017;26(5):1116-1129. doi:10.1007/s10897-017-0090-y

65. Veneris JT, Matulonis UA, Liu JF, et al. Choosing wisely: selecting PARP inhibitor combinations to promote anti-tumor immune responses beyond BRCA mutations. Gynecol Oncol. 2020;156 (2):488-497. doi:10.1016/j.ygyno.2019.09.021

66. Werner-Lin A, Hoskins LM, Doyle MH, et al. 'Cancer doesn't have an age': genetic testing and cancer risk management in BRCA1/2 mutation-positive women aged 18-24. Health. 2012;16(6):636-654. doi: $10.1177 / 1363459312442420$

67. Godina L, Turchetti T, Jackson L, et al. Impact of presymptomatic genetic testing on young adults: a systematic review. Eur J Hum. 2016;24(4):496-503. doi:10.1038/ejhg.2015.153 


\section{Publish your work in this journal}

The Application of Clinical Genetics is an international, peerreviewed open access journal that welcomes laboratory and clinical findings in the field of human genetics. Specific topics include: Population genetics; Functional genetics; Natural history of genetic disease; Management of genetic disease; Mechanisms of genetic disease;
Counselling and ethical issues; Animal models; Pharmacogenetics; Prenatal diagnosis; Dysmorphology. The manuscript management system is completely online and includes a very quick and fair peerreview system, which is all easy to use. Visit http://www.dovepress. com/testimonials.php to read real quotes from published authors. 